\title{
Deployment Kinematic Analysis and Control of a New Hoop Truss Deployable Antenna
}

\author{
Zihan Sun ${ }^{1,2,3}$, Yankang Ding ${ }^{1,2,3}$, Yiqun Zhang ${ }^{1,2,3}$, Dongwu Yang $^{12,3}$ and $\mathrm{Na} \mathrm{Li}^{1,2,3}$ \\ ${ }^{1}$ Key Laboratory of Electronic Equipment Structure Design, Ministry of Education, Xi'an, 710071, China \\ ${ }^{2}$ School of Electromechanical Engineering, Xidian University, Xi'an, 710071, China \\ ${ }^{3}$ Collaborative Innovation Center of Information Sensing and Understanding at Xidian University, Xi'an, 710071, China
}

\begin{abstract}
Firstly, based on the structural characteristics of a new type of hoop truss deployable antenna, this paper derives the motion transformation relation between two hoop modules by using the method of coordinate transformation, and establishes the general model for deployment kinematic analysis, which can be applied to analyze the position, velocity and acceleration of any point on the structure. Secondly, according to the relation between the driving cable and the hoop module, the motion planning of the hoop module is transformed into the motion control of the driving cable, which can realize the deploying position control of the antenna. Finally, numerical simulations show the control method can make the antenna smoothly deploy following the specified deployable motion.
\end{abstract}

\section{Introduction}

In the modern satellite communications, wireless broadcasting systems, earth observation and deep space exploration, large deployable antennas are one of the key equipment [1-3]. The hoop truss deployable antenna is getting more and more attention due to its superior performance. Its structure is mainly composed of deployable truss, tension supporting net, metal mesh and driving device [4]. Compared with other antenna structures, it has the advantages of large flexibility, good space thermal stability, large storage ratio, simple structure, and the increase of the aperture in a certain range does not change the structural form of the antenna, and the quality does not increase proportionally, so it is the ideal structural form for a large deployable antenna at present. However, the traditional hoop truss deployable antenna restricts its development to super-large-diameter antennas because the storage ratio is difficult to increase. Based on this, Cao, a master student of Xidian University, proposed a new structure about the hoop truss deployable antenna [5], which significantly improved the storage ratio and received great attention.

The deployable antenna has two states of folding and unfolding. In the process of satellite launching, it is in a collapsed state. After entering the orbit, the antenna automatically deploys into working state. The deploying process is one of the most brittle parts for the deployable antenna. Therefore, it is necessary to carry out the simulation of the deploying process and the prediction of the performance of the antenna $[6,7]$. The paper carried out detailed kinematics and controlling analysis by studying the new hoop truss deployable structure proposed by Cao of Xidian University.

\section{Location analysis of deployment}

A schematic view of the deploying process of hoop truss unit is shown in Fig. 1. The points $A_{i}$ to $N_{i}$ are the associate points of truss unit. The angle of $\theta$ is the deploying angle, which is the angle between the bar $A_{i} H_{i}$ and $x_{i}$ axis. In the process of deployment, point $A_{1}$ is fixed at ground and point $B_{1}$ move down along $y_{1}$ axis.

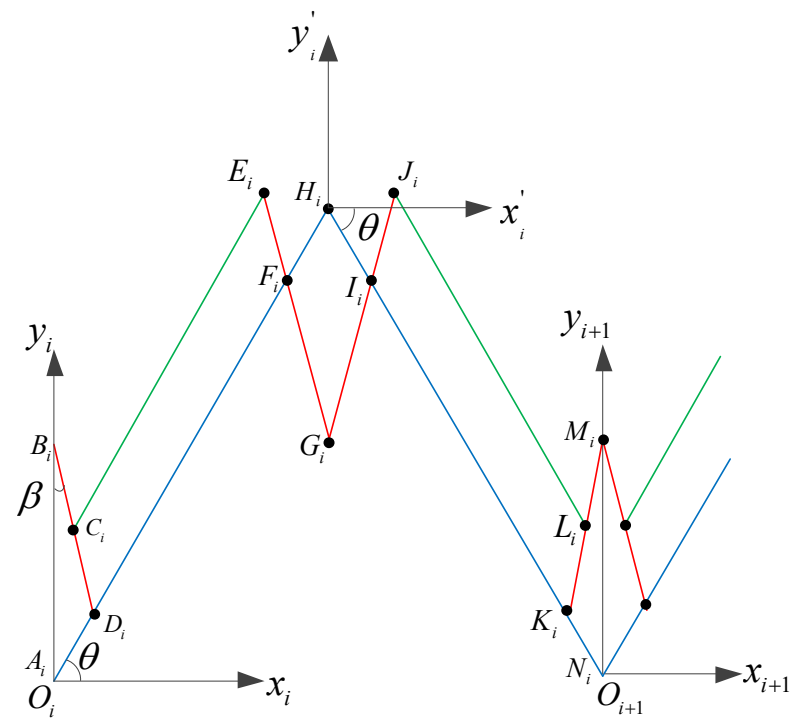

Fig.1. Truss unit coordinate system description.

In order to analyze this structure, we defined that the body coordinate system $O_{1} x_{1} y_{1} z_{1}$ is coincident with the 
inertial coordinate system $O x y z$. And $y$ axis passes through points $A_{1}$ and $B_{1}$. The angle between two $x$ axes of body coordinate system $O_{i} x_{i} y_{i} z_{i}$ and the body coordinate system $O_{i+1} x_{i+1} y_{i+1} z_{i+1}$ was defined as $\varphi$. The angle $\varphi$ was decided by truss segment number $n$, in other words, $\varphi=2 \times 360^{\circ} / n$.

In the deployment of antenna, the conversion process from the body coordinate system $O_{i} x_{i} y_{i} z_{i}$ to the body coordinate system $O_{i+1} x_{i+1} y_{i+1} z_{i+1}$ can be equivalent to shifting $O_{i} x_{i} y_{i} z_{i}$ from point $O_{i}$ to point $O_{i+1}$ firstly, and then rotating $\varphi$ around the $y$ axis in the forward direction. The transformation matrix could be obtained as

$$
{ }^{i} T_{i+1}=\left[\begin{array}{cccc}
\cos \varphi & 0 & \sin \varphi & X_{N i} \\
0 & 1 & 0 & Y_{N i} \\
-\sin \varphi & 0 & \cos \varphi & Z_{N i} \\
0 & 0 & 0 & 1
\end{array}\right]
$$

Any point $P_{i}$ can be described as $P_{i}=\left(P x_{i}, P y_{i}, P z_{i}, 1\right)^{T}$ in the body coordinate system $O_{i} x_{i} y_{i} z_{i}$, and $P_{i+1}=\left(P x_{i+1}, P y_{i+1}, P z_{i+1}, 1\right)^{T}$ in the body coordinate system $O_{i+1} x_{i+1} y_{i+1} z_{i+1}$. And both of them satisfy the conversion equation.

$$
P_{i}={ }^{i} T_{i+1} \cdot P_{i+1}
$$

During the deployment of the antenna, converting any point $P_{i}\left(A_{i} \sim N_{i}\right)$ from the body coordinate system $O_{i} x_{i} y_{i} z_{i}$ to $O_{1} x_{1} y_{1} z_{1}$ can be described as

$$
P_{1}={ }^{1} T_{i} \cdot P_{i}=\left(\prod_{k=2}^{i}{ }^{k-1} T_{k}\right) \cdot P_{i}
$$

Denote the length of $A H$ as $l_{1}$, the length of $A D$ and $F H$ as $l_{2}$, the length of $B D$ and $F G$ as $l_{3}$ and the length of $C D$ and $E F$ as $l_{4}$. The coordinate of any point on the truss could be obtained by the following four steps.

Step.1. The local coordinate system $O_{i} x_{i} y_{i} z_{i}$ is established with point $A_{i}$ on the origin. And the points $P_{i}\left(A_{i} \sim H_{i}\right)$ are in the plane $O_{i} x_{i} y_{i}$. So the coordinate value of the points $A_{i} \sim H_{i}$ can be obtained.

$$
{ }^{1} T_{i}=\left[\begin{array}{cccc}
\cos [(i-1) \varphi] & 0 & \sin [(i-1) \varphi] & \left\{\sum_{k=2}^{i}[A \cos (k-2) \varphi-B \sin (k-2) \varphi]\right\} \cos \theta \\
0 & 1 & 0 & 0 \\
-\sin [(i-1) \varphi] & 0 & \cos [(i-1) \varphi] & \left\{\sum_{k=2}^{i}[-A \sin (k-2) \varphi-B \cos (k-2) \varphi]\right\} \cos \theta \\
0 & 0 & 0 & 1
\end{array}\right]
$$

During the deployable process of antenna, the position coordinates of any point $P_{i}\left(A_{i} \sim N_{i}\right)$ in the inertial coordinate system can be calculated by the following Eq. (7).

$$
P_{1}={ }^{1} T_{i} \cdot P_{i}
$$

where $P_{i}$ is the position coordinate of the point in its body coordinate system $O_{i} x_{i} y_{i} z_{i}$.
Step.2. The local coordinate system $O_{i}^{\prime} x_{i}^{\prime} y_{i}^{\prime} z_{i}^{\prime}$ is established with point $H_{i}$ on the origin. And the points $P_{i}\left(H_{i} \sim N_{i}\right)$ are in the plane $O_{i}^{\prime} x_{i}^{\prime} y_{i}^{\prime}$. So the coordinate value of the points $P_{i}\left(H_{i} \sim N_{i}\right)$ in the plane $O_{i}^{\prime} x_{i}^{\prime} y_{i}^{\prime}$ can be obtained.

Step.3. The conversion process from the body coordinate system $O_{i} x_{i} y_{i} z_{i}$ to the body coordinate system $O_{i}^{\prime} x_{i}^{\prime} y_{i}^{\prime} z_{i}^{\prime}$ can be equivalent to shifting $O_{i} x_{i} y_{i} z_{i}$ from point $O_{i}\left(A_{i}\right)$ to point $O_{i}^{\prime}\left(H_{i}\right)$, and then rotating $\varphi$ around the $y$ axis in the forward direction. The angle of $\varphi^{\prime}$ was decided by truss segment number $n$, $\varphi^{\prime}=360^{\circ} / n$. The transformation matrix could be acquired as

$$
{ }^{i} T_{i}^{\prime}=\left[\begin{array}{cccc}
\cos \varphi & 0 & \sin \varphi^{\prime} & X_{H i} \\
0 & 1 & 0 & Y_{H i} \\
-\sin \varphi^{\prime} & 0 & \cos \varphi^{\prime} & 0 \\
0 & 0 & 0 & 1
\end{array}\right]
$$

Meanwhile, any point $P_{i}\left(H_{i} \sim N_{i}\right)$ in the body coordinate system $O_{i} x_{i} y_{i} z_{i}$ and the body coordinate system $O_{i}^{\prime} x_{i}^{\prime} y_{i}^{\prime} z_{i}^{\prime}$ can be respectively described as $P_{i}=\left(P x_{i}, P y_{i}, P z_{i}, 1\right)^{T}$ and $P_{i}^{\prime}=\left(P x_{i}^{\prime}, P y_{i}^{\prime}, P z_{i}^{\prime}, 1\right)^{T}$. And both of them satisfy the transformation equation.

$$
P_{i}={ }^{i} T_{i}^{\prime} \cdot P_{i}^{\prime}
$$

Step.4. The truss is divided into $n$ segments and $n$ must be even. The coordinate value of point $N_{i}$ is brought into Eq.(2), and then combining Eq.(2) and Eq.(3), the transformation matrix of body coordinate system $O_{i} x_{i} y_{i} z_{i}(i=2 \sim n / 2)$ to inertial coordinate system $O_{1} x_{1} y_{1} z_{1}$ can be obtained as Eq.(6). In Eq.(6), $A=l_{1}\left(1+\cos \varphi^{\prime}\right)$ and $B=l_{1} \sin \varphi^{\prime}$.

\section{Speed and acceleration analysis}

On both sides of Eq. (7), the first order derivative with respect to the time $t$ is obtained as

$$
\dot{P}_{1}={ }^{1} \dot{T}_{i} \cdot P_{i}+{ }^{1} T_{i} \cdot \dot{P}_{i}
$$


On both sides of Eq. (7), the second order derivative with respect to the time $t$ is obtained as

$$
\ddot{P}_{1}={ }^{1} \ddot{T}_{i} \cdot P_{i}+2{ }^{1} \dot{T}_{i} \cdot \dot{P}_{i}+{ }^{1} T_{i} \cdot \ddot{P}_{i}
$$

Based on Eq. (8) and Eq. (9), the velocity and acceleration values of any point on the hoop truss structure can be analyzed.

\section{Deployment and control analysis}

In the deployment of antenna, whether the deployment of angle $\theta$ is smooth or not directly determines whether there will be large vibrations. Therefore, it is necessary to plan the motion of the truss unit. The rate of change of the deploying angle can be defined as

$$
\dot{\theta}=f(t)
$$

and the integral function is obtained as

$$
\theta=\int f(t) d t+C
$$

where $C$ is a constant

Meanwhile, it is also necessary to establish the relationship between the changeable length of the driving cable and the angle in deployment of the hoop truss unit since the deployment of antenna is achieved by contracting the driving cable with a motor.

As shown in Fig. 1, the effect of the pulley radius on the change in cable length is ignored here, and considering that the driving cable generally has a good stiffness, we also ignore the effects of its force deformation. So the driving cable is similarly arranged in the mechanism at the diagonal position of the hinged four-bar units $\left(H_{i} F_{i} J_{i} I_{i}\right.$ and $\left.B_{i+1} K_{i} A_{i+1} D_{i+1}\right)$. The relationship between the length of the driving cable and the angle of deployment can be obtained from the geometric relationship (This relationship describes only kinematic relation and does not involve mechanical properties).

$$
\begin{aligned}
& L^{2}+l_{2}^{2}-2 L l_{2} \sin \theta=l_{3}^{2} \\
& \theta=\arcsin \left(\frac{L^{2}+l_{2}^{2}-l_{3}^{2}}{2 L l_{2}}\right)
\end{aligned}
$$

On both sides of Eq. (12), the first order derivative with respect to the time $t$ is obtained as

$$
\begin{gathered}
L \dot{L}-\dot{L} l_{2} \sin \theta-L l_{2} \dot{\theta} \cos \theta=0 \\
\dot{\theta}=\frac{L \dot{L}-\dot{L} l_{2} \sin \theta}{L l_{2} \cos \theta} \\
\dot{L}=\frac{L l_{2} \dot{\theta} \cos \theta}{L-l_{2} \sin \theta}
\end{gathered}
$$

On both sides of Eq. (12), the second order derivative with respect to the time $t$ is obtained as

$$
\begin{gathered}
\dot{L}^{2}+L \ddot{L}-\ddot{L} l_{2} \sin \theta-2 \dot{L} l_{2} \dot{\theta} \cos \theta \\
-L l_{2} \ddot{\theta} \cos \theta+L l_{2} \dot{\theta}^{2} \sin \theta=0 \\
\ddot{\theta}=\frac{\dot{L}^{2}+L \ddot{L}-\ddot{L} l_{2} \sin \theta-2 \dot{L} l_{2} \dot{\theta} \cos \theta+L l_{2} \dot{\theta}^{2} \sin \theta}{L l_{2} \cos \theta} \\
\ddot{L}=\frac{2 \dot{L} l_{2} \dot{\theta} \cos \theta+L l_{2} \ddot{\theta} \cos \theta-L l_{2} \dot{\theta}^{2} \sin \theta-\dot{L}^{2}}{L-l_{2} \sin \theta}
\end{gathered}
$$

So in the view of the perspective of mechanism kinematic, the corresponding relationship between the change of the length of the driving cable of the hinged four-bar units and each deploying time is obtained during the entire deploying process. When the position controlling of the deployment of antenna is performed, the linear displacement of the motor output corresponds to the frequency of the pulse input, so that the deploying process of antenna can be smoothly controlled according to the planned curve of the deploying angle.

\section{Simulation verification}

As illustrated in Fig. 2, the deployment of antenna can be generally designed as a motion which consists of acceleration stage, constant speed stage and deceleration stage.

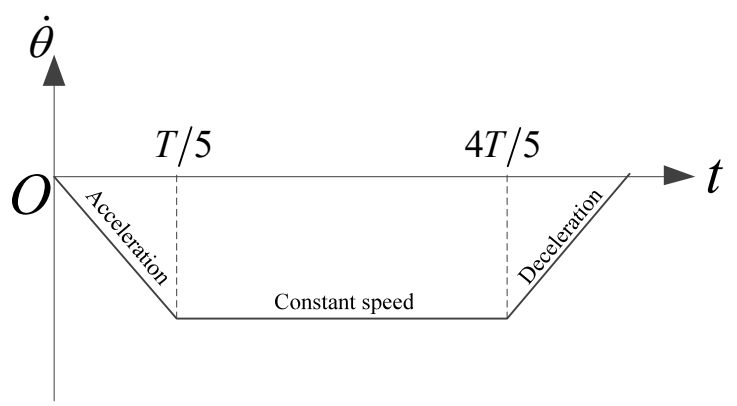

Fig. 2. Angular velocity curve.

Taking the slope of the acceleration section as $k$, the slope of deceleration section as $-k$, and the total deploying time as $T$, then the angular velocity curve is planed as

$$
\dot{\theta}= \begin{cases}k t & 0 \leq t<\frac{T}{5} \\ \frac{k T}{5} & \frac{T}{5} \leq t<\frac{4 T}{5} \\ k(T-t) & \frac{4 T}{5} \leq t \leq T\end{cases}
$$

According to the requirements of the deploying process, if $t: 0 \rightarrow T, \theta: 90^{\circ} \rightarrow 0^{\circ}$. then

$$
\theta_{\text {end }}=\int_{0}^{T / 5} k t d t+\int_{T / 5}^{4 T / 5} \frac{k T}{5} d t+\int_{4 T / 5}^{T} k(T-t) d t=-\frac{\pi}{2}
$$

the coefficient $k$ can be solved as $k=-25 \pi /\left(8 T^{2}\right)$.

Eq. (20) is integrated and the continuous angle of the deploying angle in the process is considered, so the change of the angle can be solved as

$$
\theta= \begin{cases}\frac{k}{2} t^{2}+\frac{\pi}{2} & 0 \leq t<\frac{T}{5} \\ \frac{k T}{5} t+\frac{\pi}{2}-\frac{k T^{2}}{50} & \frac{T}{5} \leq t<\frac{4 T}{5} \\ -\frac{k}{2} t^{2}+k T t+\frac{\pi}{2}-\frac{17 k T^{2}}{50} & \frac{4 T}{5} \leq t \leq T\end{cases}
$$

The ring-shaped truss deployable antenna composed of $n=12$ units with the aperture of $2 \mathrm{~m}$ and the deploying 
time $T$ of 500s is exampled here whose simulation results are shown in Figs. 3-6. It can be seen from Fig. 3 which shows the trajectory of the vertices of each rigid truss during the deployment of the antenna that the vertices on the truss are deployed along the trajectory shown by the dashed line according to the angle of deployment of planning, and the feasibility of the structure is preliminarily verified. Figs. 4-5 show the motion trajectory of unit. The trajectory is smooth, which indicates the unit can smoothly deploy. Fig. 6 shows the structural state at different times during the deploying process. It can be seen that the antenna is smoothly deployed according to the planned angular velocity variation.

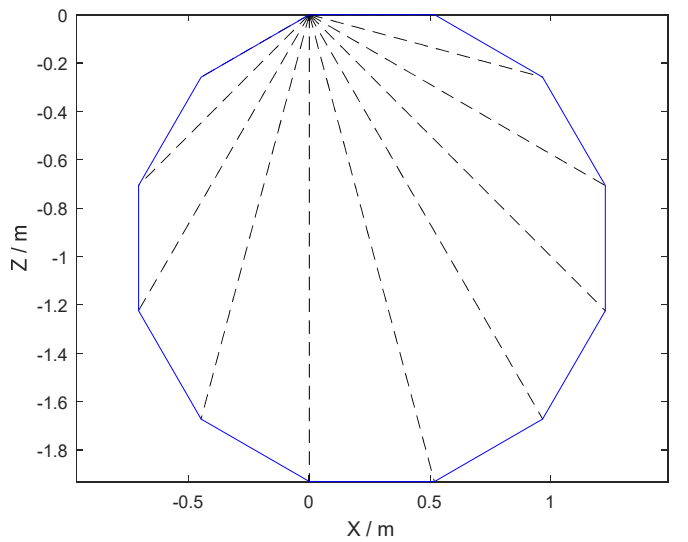

Fig.3. Trajectory of vertex $\left(A_{i}\right.$ and $\left.H_{i}\right)$.

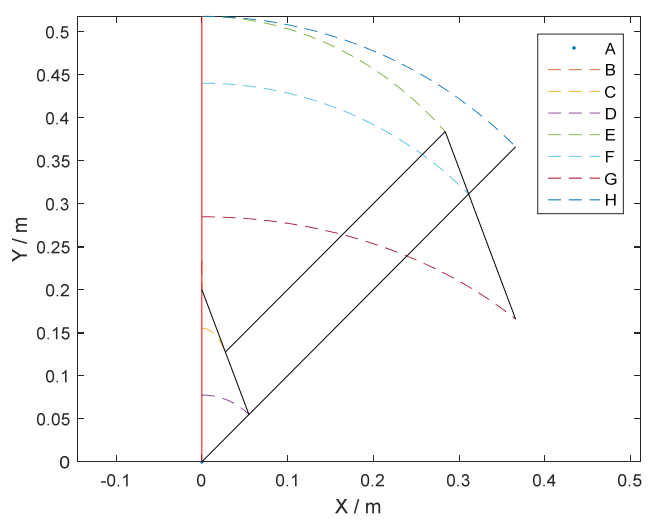

Fig.4. Unit deploying stage (node $A_{i} \sim H_{i}$ ).

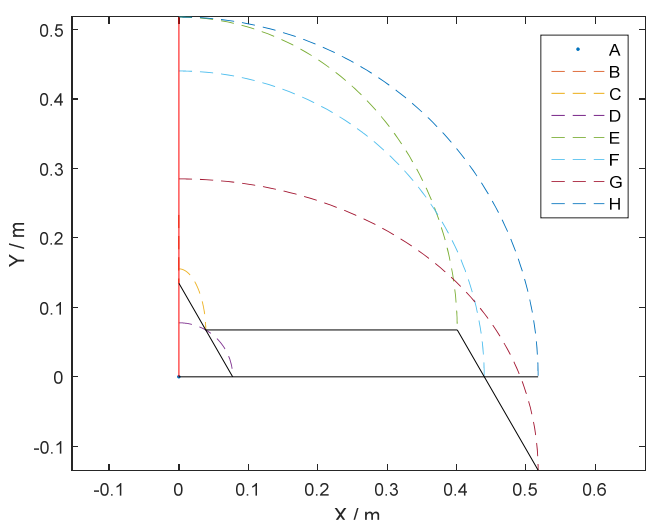

Fig. 5. Trajectory of unit nodes $\left(A_{i} \sim H_{i}\right)$.

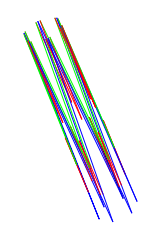

(a) $t=50 \mathrm{~s}$

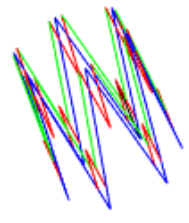

(b) $t=100 \mathrm{~s}$

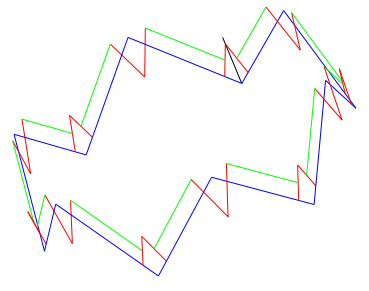

(c) $t=250 \mathrm{~s}$

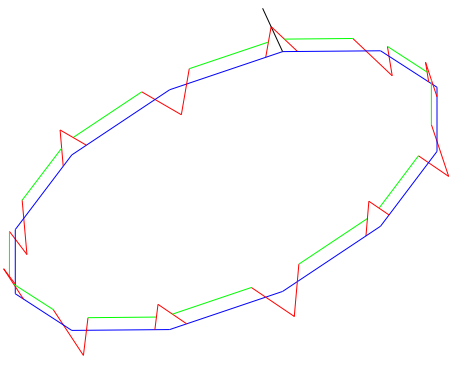

(d) $t=500 \mathrm{~s}$

Fig. 6. The analysis results of deploying process.

\section{Conclusion}

A general motion analysis model for the deployment of a new type of hoop truss deployable antenna is established. For such antennas with different structural parameters, the position, velocity and acceleration of any point on the structure can be analyzed during the deployment by using this model, so it has strong practicability. At the same time, the control strategy of deployment based on position controls is studied. On the one hand, according to the requirement of deployment, the movement planning of the unit is carried out. On the other hand, according to the structural geometry, the motion of unit is transformed into the displacement plan of the deploying device. By implementing effective motion control of the driving cable, the antenna can be smoothly deployed according to the planned variation of the angle.
This work is supported by the National Natural Science Foundation of China with No.51775404, Nature Science Basic Research Plan in Shaanxi Province with No. 2016JQ5072 \& No. 2016JQ5006, Fundamental Research Funds for the Central Universities with No.JB180410, and the Shanghai Aerospace Science and Technology Innovation Fund.

\section{Reference}

1. Clasen G, Langley R. Meshed patch antennas[J]. IEEE Transactions on Antennas and Propagation, 52(6):1412-1416, (2004)

2. Terada M, Bludworth N, Moore J, et al. Deployable reflector system for satellite applications[C]// Sbmo/ieee Mtt-S International Conference on Microwave and Optoelectronics. IEEE, p.647-649, (2005) 
3. Zhang J G, Chen J J, Duan B Y. Reliability analysis of the deployment mechanism of a large satellite antenna based on the non-probabilistic model[J]. Journal of Xidian University, 33(5):739-744, (2006). (in Chinese)

4. Thomson M W. The Astromesh deployable reflector[C]// Antennas and Propagation Society International Symposium[C]. IEEE, p.1516-1519, (2000)
5. Cao Peng, Bao Hong, Duan Baoyan, Zhang Yiqun, Expandable mechanism of space plane thin film antenna. CN,201710607804.1, (2017). (in Chinese)

6. Li Tuan-Jie, Zhang Y, Duan B Y, Deployment kinematic analysis and control of hoop truss deployable antenna[J]. Journal of Xidian University, 34(6):916-921, (2007). (in Chinese)

7. Duan Baoyan. Flexible antenna structure analysis, optimization and control. Beijing: Science Press, p.346-362, (2005). (in Chinese) 